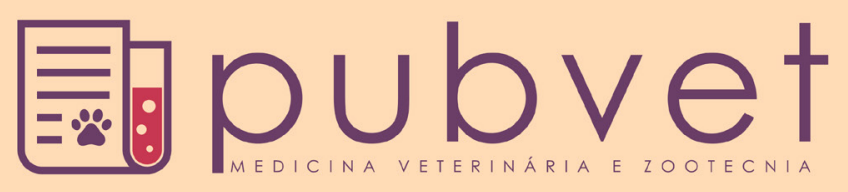

HTTP://DX.DOI.ORG/10.22256/PUBVET.V11N7.701-704

\title{
Quimiodectoma maligno em um cão Weimaraner: Relato de caso
}

\author{
Guilherme Dias Araujo $^{1 *}$, Tais Meziara Wilson ${ }^{2}$, Daniela Marques Bernardo ${ }^{3}$, Marco \\ Túlio Gomes Campos ${ }^{1}$, Diogo Nogueira Araújo ${ }^{1}$
}

${ }^{I}$ Médico Veterinário, Universidade de Uberaba (Uniube) (Uberaba-MG, Brasil).

${ }^{2}$ Médica Veterinária, Universidade Federal de Uberlândia (UFU) (Uberlândia-MG, Brasil).

${ }^{3}$ Médica Veterinária, Pontificia Universidade Católica de Minas Gerais (PUC) (Poços de Caldas-MG, Brasil).

*Autor para correspondencia, E-mail: E-mail: guilherme.d.a@bol.com.br

RESUMO. O presente relato de caso tem como finalidade descrever uma ocorrência de quimiodectoma de caráter maligno em um cão de raça Weimaraner. Quimiodectomas são neoplasias formadas a partir de quimiorreceptores presentes na base do coração envolvendo o corpo aórtico e carótido, pode ser benigno (mais comum) ou maligno. Estes tumores são pouco frequentes na clínica de pequenos animais e, quando ocorrem, geralmente estão associados a cães idosos e de raças braquiocefálicas (boxer, bulldog, dentre outros). $\mathrm{O}$ diagnóstico clínico é dificultoso, pois a maioria dos animais acometidos é assintomática, e, quando presentes, os sinais clínicos são inespecíficos, sendo, portanto, diagnosticados através dos exames radiográficos de rotina ou como achados nos exames post-mortem. No presente caso, o animal foi a atendimento clínico por outro motivo (nódulos subcutâneos), tendo sido investigado o quimiodectoma após ser percebido aumento de frequência respiratória e tamponamento de sons pulmonares em algumas regiões torácicas. $\mathrm{O}$ diagnóstico final foi possível após radiografia torácica e histopatologia do tecido tumoral através de técnica "tru-cut" com auxílio de ultrasonografia com ferramenta Doppler. Além de se referir à ocorrência de quimiodectoma em uma raça não predisposta, a importância do presente trabalho também se dá à abordagem do proprietário frente a uma patologia crônica maligna sem possibilidade de correção.

Palavras chave: Quimiodectoma, neoplasias caninas

\section{Malignant chemokidoma in a Weimaraner dog: Case report}

ABSTRACT. The goal of this case report is to describe an occurrence of malginant chemodectoma in a weimaraner dog. Chemodectomas are tumors formed from chemoreceptors present at the heart base involving the aortic and carotid body, can be benign (most common) or malignant. These tumors are uncommon in small animal clinical and, when they occur, are usually associated with older dogs and brachiocephalic breeds (boxer, bulldog, among others). The clinical diagnosis is cornered, because the most of affected animals are asymptomatic, and when present, the clinical signs are non-specific and, therefore, diagnosed through routine radiographs or as found in post-mortem examination. In this case, the animal went to clinical treatment for some other reason (subcutaneous nodules), and the chemodectoma was investigated after be perceived increased respiration and buffering lung sounds in some thoracic regions. The final diagnosis was possible after thoracic radiography and histopathology of tumor tissue through "tru-cut" technique with the aid of ultrasound Doppler tool. In addition to refer to the occurrence of chemodectoma in a non-biased race, the importance of this work is also about gives the owner approach against a malignant chronic pathology without possibility of correction.

Keywords: Chemodectoma, canine neoplasias 


\section{Quimiodectoma maligno en un perro Weimaraner: Reporte de un caso}

RESUMEN. El presente relato de caso tiene como propósito describir una ocurrencia de quimiodectoma de carácter maligno en un perro de raza Weimaraner. Los quimiodectomas son neoplasias formadas a partir de quimiorreceptores presentes en la base del corazón envolviendo el cuerpo aórtico y carótido, puede ser benigno (más común) o maligno. Estos tumores son poco frecuentes en la clínica de pequeños animales $\mathrm{y}$, cuando ocurren, generalmente están asociados a perros de edad avanzada y de razas braquiocefálicas (Boxer, Bulldog, entre otros). El diagnóstico clínico es difícil, ya que la mayoría de los animales afectados son asintomáticos, y cuando están presentes, los signos clínicos son inespecíficos y, por lo tanto, se diagnostican a través de los exámenes radiográficos de rutina o en los exámenes post-mortem. En el presente caso, el animal fue llevado para consulta clínica por otro motivo (nódulos subcutáneos), siendo investigado el quimiodectoma tras ser percibido aumento de frecuencia respiratoria y taponamiento de sonidos pulmonares en algunas regiones torácicas. El diagnóstico final fue posible después de la radiografía torácica e histopatología del tejido tumoral a través de técnica tru-cut con auxilio de ultrasonografía con herramienta Doppler. Además de referirse a la ocurrencia de quimiodectoma en una raza no predispuesta, la importancia del presente trabajo también se da al abordaje del propietario frente a una patología crónica maligna sin posibilidad de corrección.

Palabras clave: canino, neoplasia, quimiorreceptores

\section{Introdução}

Os quimiorreceptores localizados na base do coração envolvendo o corpo aórtico e carótido, são responsáveis pela monitoração dos níveis sanguíneos de oxigênio, dióxido de carbono, temperatura e $\mathrm{pH}$, onde exercem função moduladora do sistema cardiorespiratório quando ocorrem alterações nos parâmetros físiológicos (Sampaio et al., 2008). Os tumores oriundos desses receptores sensoriais são chamados de quimiodectomas, sendo considerados tumores primários de base cardíaca, já que, apesar de serem formados por tecidos extracardíacos, são responsáveis por transtornos cardiovasculares (Garrido et al., 2008).

Apesar de serem pouco frequentes na clínica de pequenos animais, os quimiodectomas são os tumores cardíacos mais comuns em cães depois do hemangiossarcoma cardíaco, podendo ser malignos ou, mais comumente, benignos (Mesquita et al., 2012).

O diagnóstico clínico é dificultoso, pois a maioria dos animais acometidos é assintomático. Quando os sinais clínicos são presentes, compreendem alterações características de insuficiência cardíaca congestiva aguda, como tosse, dispneia, cianose, ascite, hidrotórax, hidropericádio, anasarca, congestão pulmonar, congestão hepática, dentre outros. Também há possibilidade de o animal apresentar morte súbita, sem sinais clínicos anteriores, devido choque cardiogênico agudo. Portanto, os quimiodectomas são diagnosticados através dos exames radiográficos de rotina (seguidos de exames mais específicos do sistema cardiovascular) ou como achados nos exames post-mortem (Ferreira et al., 2010, Garrido et al., 2008).

O tratamento cirúrgico do tumor geralmente é inviável, devido a sua localização e estruturas anatômicas relacionadas, e/ou, adjacentes à sua massa (Salomão et al., 2012). Dessa forma, a orientação adequada ao proprietário deve ser realizada, explicitando corretamente as alterações patológicas que o animal possa sofrer, e o prognóstico ruim da enfermidade.

$\mathrm{O}$ presente relato de caso tem como objetivo descrever os achados clinicopatológicos de quimiodectoma maligno em um cão weimaraner.

\section{Relato de caso}

Um cão, fêmea, da raça weimaraner, $30 \mathrm{~kg}$, de nove anos de idade, deu entrada no Hospital Veterinário de Uberaba apresentando como queixa dois tumores subcutâneos.

Ao exame clínico geral e especial foram constatados dois tumores subcutâneos, não ulcerados, não aderidos, de superfície regular à 
palpação, medindo $5 \times 6 \times 2 \mathrm{~cm}$ e $4 \times 6 \times 1 \mathrm{~cm}$, respectivamente, em região torácica lateral direita e flanco direito. Além disso, o animal apresentava mucosas levemente hipocoradas, taquipnéia (68 movimentos respiratórios/minuto) e ausculta pulmonar possibilitada apenas em focos mais dorsais e caudais de ambos hemitórax. Também havia tamponamento de alguns focos de ausculta cardíaca principalmente em base direita, embora a frequência cardíaca conferida pelo pulso femoral se mantivesse estável ( 88 batimentos/minuto).

Frente às alterações notadas, foi solicitado o exame radiológico da região torácica para esclarecimento de possível alteração cardíaca e pulmonar, assim como, pesquisa de possível metástase pulmonar causada pelos tumores subcutâneos antes de decidir sobre exerese cirúrgica.

Como achado ocasional no exame radiológico torácico, foi caracterizada uma cardiomegalia acentuada e presença de massa torácica de aspecto denso e nodular, com radiopacidade de tecidos moles, formato arredondado, dimensões de aproximadamente $14,5 \mathrm{~cm}$ (largura) x $10 \mathrm{~cm}$ (altura), sobreposta à silhueta cardíaca em sua porção médio-cranial, e aos terceiros, quartos, quintos e sextos pares de costelas e as terceira, quarta, quinta e sexta esternébras, em projeção látero-lateral direita.

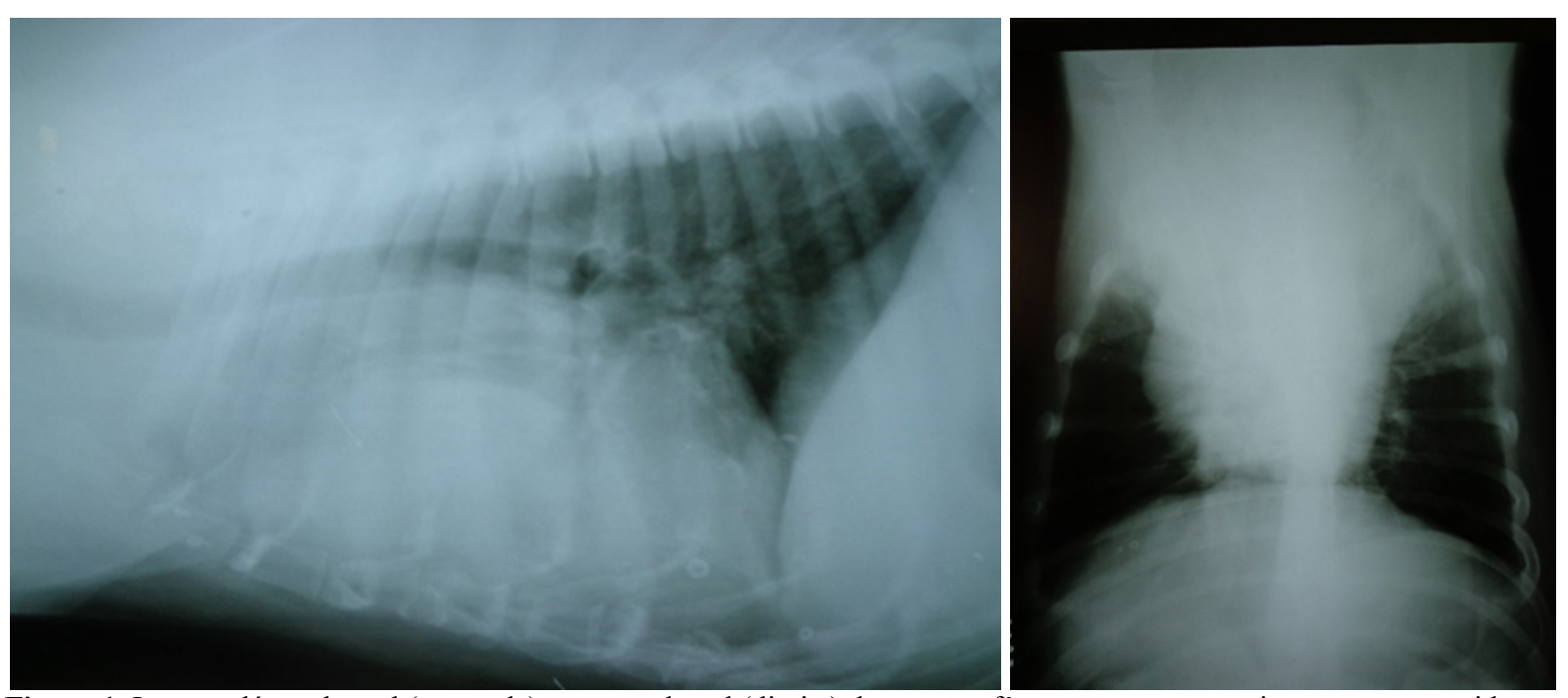

Figura 1. Imagem látero-lateral (esquerda) e ventro-dorsal (direita) de um cão, fêmea, nove anos, weimaraner, acometido por quimiodectoma maligno. Notar a massa arredondada, radiopaca, densa e nodular cranialmente à base do coração.

Pela sugestão de tumor intratorácico explicitado pelo exame radiológico, o animal foi encaminhado para realização de exame histopatológico da estrutura. Dois fragmentos, medindo em conjunto, $1,2 \times 0,2 \times 0,1 \mathrm{~cm}$, da amostra foram coletados através de biópsia tru-cut auxiliado por ultrassonografia com ferramenta Doppler. Macroscopicamente os fragmentos apresentavam aspecto esbranquiçado e microscopicamente a análise evidenciou neoplasia maligna caracterizada por células redondas agrupadas em ninhos e esboços de rosetas, com núcleos amplos, hipercorados, citoplasma vacuolizado, pálido, esparsas figuras de mitose e estroma fibroso. Diagnóstico de carcinoma neuroendócrino morfologicamente sugestivo de quimiodectoma ou paraganglioma.

Foram solicitados ao proprietário outros exames complementares, como eletrocardiograma, ecodopplercardiograma, e imunohistoquímica, para estabelecer a severidade e natureza do processo, porém não foram autorizados.

O proprietário foi instruído a manter o animal em repouso e observá-lo, principalmente, quanto à função respiratória. Foi explicada a inviabilidade do procedimento cirúrgico, pela localização do tumor e as importantes estruturas anatômicas relacionadas, bem como a possibilidade de o animal sofrer morte súbita insuficiência cardíaca e respiratória.

\section{Discussão e Conclusão}

Apesar de o caso relatado se referir a um quimiodectoma em cão de raça weimaraner, essas neoplasias geralmente são mais encontradas em cães de raça braquiocefálica, principalmente na raça boxer (Moura et al., 2006, Garrido et al., 2008). Entretanto, a determinada raça é de grande 
porte, em acordo com os casos relatados em outros cães de mesmo porte, como Fila Brasileiro e Rotwailler relatados por Sampaio et al. (2008). Poucos casos de quimiodectomas são diagnosticados em cães de pequeno porte como o relatado por Salomão et al. (2012) em um cão da raça poodle.

Evidenciado de forma acidental no determinado caso, quimiodectomas geralmente não geram sinais clínicos que sejam perceptíveis ao proprietário, em muitos casos, gerando morte súbita e sendo diagnosticados apenas no postmortem, como relatado por Moura et al. (2006). Em outros casos, sintomatologias podem surtir em consequência do crescimento do tumor. Ferreira et al. (2010) relataram um caso de fila brasileiro apresentando sinais clínicos característicos de Síndrome da Veia Cava Cranial (SVCC) secundária a massa de um quimiodectoma invadindo a luz do átrio esquerdo do coração do animal.

Assim como há necessidade de realização de exame histopatológico para diagnóstico definitivo do tipo de tumor, também seria interessante a realização de exame imunohistoquímico para diferenciação histológica distinta de neoplasias que possam apresentar o mesmo padrão histológico (Noszczyk-Nowak et al., 2010). Além disso, exames cardiológicos específicos, com eletrocardiograma e ecocardiograma, também se fazem necessário para pesquisa de disfunções elétricas e morfológicas do coração. Cavalcanti et al. (2006) relataram disfunções elétricas de despolarização atrial em cão, sem raça definida, acometido por quimiodectoma.

Apesar de não ser uma enfermidade recorrente na rotina clínica de cães, o quimiodectoma deve ser reconhecido como diagnóstico diferencial de cães apresentando sinais de insuficiência respiratória e cardíaca, mesmo que não seja de raças braquiocefálicas. O diagnóstico correto se faz necessário, principalmente, para a conscientização do proprietário acerca da possível evolução clínica da patologia e de seu prognóstico.

\section{Referências bibliográficas}

Cavalcanti, G. A. O., Muzzi, R. A. L., Bezerra Júnior, P. S., Nogueira, R. B. \& Varaschin, M. S. 2006. Fibrilação atrial em cão associada ao quimiodectoma infiltrativo atrial: relato de caso. Arquivo Brasileiro de Medicina Veterinária e Zootecnia, 58, 1043-1047.
Ferreira, F. S., Silveira, L. L., Vale, D. F., Salavessa, C. M., Barretto, F. L., Oliveira, A. L. A., Antunes, F., Queiroz, E. C. \& Carvalho, C. B. 2010. Síndrome da veia cava cranial (SVCC) secundária a quimiodectoma aórtico em cão-relato de caso Cranial vena cava syndrome secondary to aortic chemodectoma in dog. Revista Portuguesa de Ciencias Veterinarias, 109, 573-576.

Garrido, E., Jacintho, A. P. P., Magalhães, G. M., Cândido, E. M. \& Vasconcellos, R. O. 2008. Quimiodectoma em cão-relato de caso. Veterinária e Zootecnia, 15, 4950.

Mesquita, L. P., Abreu, C. C., Nogueira, C. I., Wouters, A. T. B., Wouters, F., Júnior, P. S. B., Muzzi, R. A. L. \& Varaschin, M. S. 2012. Prevalência e aspectos anatomopatológicos das neoplasias primárias do coração, de tecidos da base do coração e metastáticas, em cães do Sul de Minas Gerais (1994-2009). Pesquisa Veterinaria Brasileira, 32, 1155-1163.

Moura, V. M. B. D., Goiozo, P. F. I., Thomé, H. E., Caldeira, C. P. \& Bandarra, E. P. 2006. Quimiodectoma como causa de morte súbita em cão-relato de caso. Veterinária Notícias, 12, 95-99.

Noszczyk-Nowak, A., Nowak, M., Paslawska, U., Atamaniuk, W. \& Nicpon, J. 2010. Cases with manifestation of chemodectoma diagnosed in dogs in Department of Internal Diseases with Horses, Dogs and Cats Clinic, Veterinary Medicine Faculty, University Environmental and Life Sciences, Wroclaw, Poland. Acta Veterinaria Scandinavica, 52, 1-7.

Salomão, M. C., Mattos, A. S., Lucena, A. R., Leite, J. d. S., Mello, M. F. V. \& Ferreira, A. M. R. 2012. Tumor de arco aórtico em cão (Canis familiaris)-relato de caso. Veterinária e Zootecnia, 19, 107-109.

Sampaio, R. L., Coelho, H. E., Lacerda, M. S., Oliveira, P. L., Paro, P. Z., Rezende, R. S. \& Bittar, J. F. F. 2008. Efusão pleural secundária por tumor de corpo aórtico em cães-relato de caso. Veterinária Notícias, 14, 55-62.

\section{Article History:}

Received 4 March 2017

Accepted 30 April 2017

Available on line 11 June 2017

License information: This is an open-access article distributed under the terms of the Creative Commons Attribution License 4.0, which permits unrestricted use, distribution, and reproduction in any medium, provided the original work is properly cited. 